\title{
New Solutions for the Generalized BBM Equation in terms of Jacobi and Weierstrass Elliptic Functions
}

\author{
Alvaro H. Salas $\mathbb{D},{ }^{1}$ Lorenzo J. Martinez $H \mathbb{D}^{2}{ }^{2}$ and David L. Ocampo R $\mathbb{D}^{2}$ \\ ${ }^{1}$ Department of Mathematics and Statistics, FIZMAKO Research Group, Universidad Nacional de Colombia, Nubia Campus, \\ Manizales, Caldas, Colombia \\ ${ }^{2}$ Department of Mathematics, Department of Mathematics and Statistics, Universidad de Caldas, Universidad Nacional de Colombia, \\ Nubia Campus, Manizales, Caldas, Colombia
}

Correspondence should be addressed to Alvaro H. Salas; ahsalass@unal.edu.co

Received 6 January 2021; Revised 30 March 2021; Accepted 8 April 2021; Published 23 April 2021

Academic Editor: J.-C. Cortés

Copyright ( 2021 Alvaro H. Salas et al. This is an open access article distributed under the Creative Commons Attribution License, which permits unrestricted use, distribution, and reproduction in any medium, provided the original work is properly cited.

The Jacobi elliptic function method is applied to solve the generalized Benjamin-Bona-Mahony equation (BBM). Periodic and soliton solutions are formally derived in a general form. Some particular cases are considered. A power series method is also applied in some particular cases. Some solutions are expressed in terms of the Weierstrass elliptic function.

\section{Introduction}

The regularized long-wave (RLW) equation is a famous nonlinear wave equation which gives the phenomena of dispersion and weak nonlinearity, including magneto hydrodynamic wave in plasma, phonon packets in nonlinear crystals, and nonlinear transverse waves in shallow water or in ion acoustic. This equation is also called the BBM (BenjaminBona-Mahony) equation and reads

$$
u_{t}+u u_{x}+u_{x}-\mu u_{x x t}=0 .
$$

It describes approximately the unidirectional propagation of long waves in certain nonlinear dispersive systems, and it was proposed by Benjamin et al. in 1972 [1] as a more satisfactory model than the $\mathrm{KdV}$ equation [2]:

$$
u_{t}+u u_{x}+u_{x x x}=0 .
$$

It is easy to see that Equation (1) can be derived from the equal width equation [3]:

$$
u_{t}+u u_{x}-\mu u_{x x t}=0
$$

by means of the change of variable $u \longleftarrow u+1$, that is, by replacing $u$ with $u+1$. This last equation is considered an equally valid and accurate model for the same wave phenomena simulated by (1) and (2). On the other hand, some researches analyzed the generalized $\mathrm{KdV}$ equation with variable coefficients

$$
u_{t}+\sigma u^{n} u_{x}+\mu u_{x x t}=0
$$

because this model has important applications in several fields of science. Motivated by these facts, we will consider here the generalized equal width (RW) equation with constant coefficients

$$
u_{t}+\sigma u^{n} u_{x}-\mu u_{x x t}=0
$$

Our aim is to present solutions different from those in $[2,4-6]$.

1.1. Trigonometric and Soliton Solutions. In order to obtain new solutions to Equation (5), we make the traveling wave transformation

$$
u(x, t)=u(\xi), \text { where } \xi=x-\lambda t
$$


Inserting (6) into (5) gives

$$
-\lambda u^{\prime}(\xi)+u(\xi)^{n} \sigma u^{\prime}(\xi)+\lambda \mu u^{\prime \prime \prime}(\xi)=0
$$

Integrating once with respect to $\xi$ to get

$$
-\lambda u(\xi)+\frac{\sigma}{n+1} u^{n+1}(\xi)+\lambda \mu u^{\prime \prime}(\xi)-c_{1}=0
$$

where $c_{1}$ is the constant of integration. Now, we multiply Equation (8) by $u^{\prime}(\xi)$, and we integrate the resulting equation with respect to $\xi$ :

$-\frac{c_{1}^{2}}{2 \lambda}-c_{1} u(\xi)+c_{2}+\frac{\sigma u(\xi)^{n+2}}{(n+1)(n+2)}+\frac{1}{2} \mu u^{\prime}(\xi)^{2}-\frac{1}{2} \lambda u(\xi)^{2}=0$,

where $c_{2}$ is the constant of integration. If $c_{1}=c_{2}=0$, Equation (9) takes the form

$$
\frac{\mu}{2} u^{2}(\xi)+\frac{\sigma}{(n+1)(n+2)} u^{n+2}(\xi)-\frac{\lambda}{2} u^{\prime}(\xi)^{2}=0
$$

Let

$$
u(\xi)=v^{r}(\xi), r v(\xi) \neq 0
$$

Inserting Equation (11) into (10), we obtain

$$
v^{\prime}(\xi)^{2}=\frac{\lambda v(\xi)^{2}}{\mu r^{2}}-\frac{2 \sigma v(\xi)^{n r+2}}{\mu(n+1)(n+2) r^{2}}
$$

From Equation (12), if $r=1 / n$, then

$$
v^{\prime}(\xi)^{2}=\frac{\lambda n^{2} v(\xi)^{2}}{\mu}-\frac{2 n^{2} \sigma v(\xi)^{3}}{\mu(n+1)(n+2)} .
$$

A sech solution to Equation (13) is

$v(\xi)=\frac{\lambda(n+1)(n+2)}{2 \sigma} \operatorname{sech}^{2}\left(\frac{n}{2} \sqrt{\frac{\lambda}{\mu}}\left(\xi+\xi_{0}\right)\right), \quad \xi_{0}=$ const.

It is clear that function

$$
u(x, t)=\sqrt[n]{\frac{\lambda(n+1)(n+2)}{2 \sigma} \operatorname{sech}^{2}\left(\frac{n}{2} \sqrt{\frac{\lambda}{\mu}\left(x-\lambda t+\xi_{0}\right)}\right)}, \quad \lambda>0
$$

is a solution to equation $u_{t}+\sigma u^{n} u_{x}-\mu u_{x x t}=0$ for any choice of the parameters $\lambda$ and $\xi_{0}$.
Finally, if $\mu<0$, then taking into account that

$$
\operatorname{sech}\left(\frac{n}{2} \sqrt{\frac{\lambda}{\mu}}\left(x-\lambda t+\xi_{0}\right)\right)=\sec \left(\frac{n}{2} \sqrt{\frac{\lambda}{-\mu}}\left(x-\lambda t+\xi_{0}\right)\right) \text {, }
$$

we obtain from (15) the trigonometric solution

$$
u(x, t)=\sqrt[n]{\frac{\lambda(n+1)(n+2)}{2 \sigma} \sec ^{2}\left(\frac{n}{2} \sqrt{\frac{\lambda}{-\mu}}\left(x-\lambda t+\xi_{0}\right)\right)} .
$$

\section{The Novel Solutions}

In this section, we will give analytical solutions to Equation (5) for two special cases: $n=1$ and $n=2$. First of all, observe that Equation (5) is equivalent to Equation (9).

2.1. The Case $n=1$. If $n=1$, Equation (9) reads

$$
\frac{\lambda}{2} u^{2}(\xi)+\frac{\sigma}{6} u^{3}(\xi)-\frac{\lambda \mu}{2} u^{\prime}(\xi)^{2}+c_{1} u(\xi)+c_{2}=0
$$

To solve this equation, we consider the reduced Duffing equation

$$
y^{\prime \prime}(\xi)=q y^{3}(\xi), \quad q \neq 0,
$$

where the coefficient $q$ does not depend on $\xi$. Observe that

$$
y^{\prime}(\xi) y^{\prime \prime}(\xi)=y^{\prime}(\xi) q y^{3}(\xi)
$$

and integrating this equation w.r.t. $\xi$ gives

$$
\frac{1}{2} y^{\prime}(\xi)^{2}=\frac{q}{4} y^{4}(\xi)+c_{3}
$$
let

Suppose that $y=y(\xi)$ is a solution to Equation (19) and

$$
u(\xi)=A y^{2}(\xi)+\frac{\lambda}{\sigma}, \text { where } A \text { is some constant }
$$

We have

$$
\begin{aligned}
u^{\prime}(\xi) & =2 A y(\xi) y^{\prime}(\xi), \\
u^{\prime \prime}(\xi) & =2 A y^{\prime}(\xi)^{2}+2 A y(\xi) y^{\prime \prime}(\xi), \\
y^{\prime}(\xi)^{2} & =\frac{q}{2} y(\xi)^{4}+2 c_{3} .
\end{aligned}
$$

Taking into account Equations (18) and (23), we must have 


$$
\begin{aligned}
& \left(-A^{2} q \lambda \mu+\frac{1}{6} A^{3} \sigma\right) y(\xi)^{6}+\left(A c_{1}-2 A^{2} c_{3} \lambda \mu-\frac{A \lambda^{2}}{2 \sigma}\right) y(\xi)^{2} \\
& +c_{2}-\frac{\lambda^{3}}{3 \sigma^{2}}+\frac{\lambda c_{1}}{\sigma}=0 .
\end{aligned}
$$

Equating to zero the coefficients of $y^{6}, y^{2}$, and $y^{0}$, we conclude that

$$
\begin{aligned}
q & =\frac{A \sigma}{6 \lambda \mu}, \\
c_{3} & =\frac{2 c_{1} \sigma-\lambda^{2}}{4 A \lambda \mu \sigma} .
\end{aligned}
$$
ODE

We have proved that the general solution to the nonlinear

$$
\frac{\lambda}{2} u^{2}(\xi)+\frac{\sigma}{6} u^{3}(\xi)-\frac{\lambda \mu}{2} u^{\prime}(\xi)^{2}+c_{1} u(\xi)+c_{2}=0
$$

may be written in the form $u(\xi)=A y^{2}(\xi)+\lambda / \sigma$, where $y=y$ $(\xi)$ is a solution to the Duffing equation

$$
y^{\prime \prime}(\xi)=\frac{A \sigma}{6 \lambda \mu} y^{3}(\xi), \text { with } y^{\prime}(\xi)^{2}=\frac{A \sigma}{12 \lambda \mu} y(\xi)^{4}+\frac{2 c_{1} \sigma-\lambda^{2}}{2 A \lambda \mu \sigma} .
$$

According to [2], the general solution to Equation (29) may be expressed in terms of the Jacobian elliptic functions. More exactly, the general solution to Equation (19) is

$$
y(\xi)=y_{0} \mathrm{cn}\left(\sqrt{\frac{A \sigma y_{0}^{2}}{6 \lambda \mu}}\left(\xi+\xi_{0}\right), \frac{\sqrt{2}}{2}\right)+\frac{\lambda}{\sigma}, \text { where } q y_{0}^{2} \neq 0 .
$$

The value of $\xi_{0}$ is determined by solving equation $y^{\prime}(0)$ $=y_{1}$. In principle, we may consider that $\xi_{0}$ is any number as well as $y_{0}$. Taking into account these facts, we have proved that the general solution to Equation (28) is

$$
u(\xi)=y_{0}^{2} \mathrm{cn}^{2}\left(\sqrt{\frac{A \sigma y_{0}^{2}}{6 \lambda \mu}}\left(\xi+\xi_{0}\right), \frac{\sqrt{2}}{2}\right)+\frac{\lambda}{\sigma}, \quad y_{0} \neq 0 .
$$

Finally, an exact solution to the EW-equation $u_{t}+\sigma u u_{x}$ $-\mu u_{x x t}=0$ is

$$
u(x, t)=A y_{0}^{2} \mathrm{cn}^{2}\left(\sqrt{\frac{A \sigma y_{0}^{2}}{6 \lambda \mu}}\left(x-\lambda t+\xi_{0}\right), \frac{\sqrt{2}}{2}\right)+\frac{\lambda}{\sigma} .
$$

Solution (32) involves four arbitrary constants: $A, y_{0}, \lambda$, and $\xi_{0}$ (these do not depend on $\xi$ ). We choose $A$ so that $A \sigma$ $y_{0}^{2} / 6 \lambda \mu>0$.
2.2. The Case $n=2$. This case corresponds to the so-called modified BBM or modified equal width equation. When $n$ $=2$, Equation (9) reads

$$
\frac{\lambda}{2} u^{2}(\xi)+\frac{\sigma}{12} u^{4}(\xi)-\frac{\lambda \mu}{2} u^{\prime}(\xi)^{2}+c_{1} u(\xi)+c_{2}=0 .
$$

This equation is harder to solve. We seek solution to Equation (33) in the form

$$
u(\xi)=p+\frac{q}{1+\sqrt{r} \operatorname{cn}\left(\sqrt{w}\left(\xi-\xi_{0}\right), \sqrt{m}\right)} .
$$

Observe that function $y=y(\xi)=r \operatorname{cn}\left(\sqrt{w}\left(\xi+\xi_{0}\right), \sqrt{m}\right)$ satisfies the nonlinear differential equation

$$
y^{\prime \prime}+w(1-2 m) y+\frac{2 m w}{r} y^{3}=0,
$$

for any constants $\gamma$ and $\xi_{0}$. This equation is Duffing equation $y^{\prime \prime}+\alpha y+\beta y^{3}=0$ with coefficients $\alpha$ and $\beta$. The relationship between the frequency $\omega$, the modulus $m$, and the coefficients is given by the system

$$
\begin{gathered}
\alpha=w(1-2 m), \\
\beta=\frac{2 m w}{r} .
\end{gathered}
$$

Solving this system for $\omega$ and $m$ gives

$$
\begin{gathered}
\omega=\alpha+r \beta, \\
m=\frac{r \beta}{2(\alpha+r \beta)}=\frac{r \beta}{2 w} .
\end{gathered}
$$

To solve the modified BBM equation, we will make use of the power series method (PSM). This is a promising method that also may be applicable to obtain an approximate solution for those ODE's that do not admit solution in closed form. In our case, we consider the function

$$
F(\xi)=\frac{\lambda}{2} u^{2}(\xi)+\frac{\sigma}{12} u^{4}(\xi)-\frac{\lambda \mu}{2} u^{\prime}(\xi)^{2}+c_{1} u(\xi)+c_{2},
$$

where

$$
u(\xi)=p+\frac{q}{1+\sqrt{r} \operatorname{cn}\left(\sqrt{w}\left(\xi-\xi_{0}\right), \sqrt{m}\right)} .
$$

The values of $q$ and $r$ are obtained from the initial conditions $u\left(\xi_{0}\right)=0$ and $u\left(\xi_{0}+K(\sqrt{m}) / \sqrt{w}\right)=0$, that is,

$$
\begin{aligned}
& p+\frac{q}{1+\sqrt{r}}=1, \\
& p+\frac{q}{1-\sqrt{r}}=0 .
\end{aligned}
$$


Thus,

$$
\begin{aligned}
\sqrt{r} & =\frac{1}{2 p-1}, \\
q & =\frac{2 p(p-1)}{2 p-1} .
\end{aligned}
$$

Inserting the ansatz (39) into (38), we obtain an expression for $F(\xi)$ in terms of the unknowns $w, m, p$, and $\lambda$. Observe that function $u(\xi)$ given by (39) is a solution to Equation (33), if and only if $F(\xi) \equiv 0$. The series method consists in coefficients of Taylor series of $F(\xi)$ around some point. Usually, this is the origin. Since the calculations are enormous, we cannot realize this work by hand. Instead, we may use Mathematica 12 or Maple 16. The Mathematica command has the form Series $\left[F[\xi],\left\{\xi, \xi_{0}, n\right\}\right]$. This gives us the $n$th degree Taylor polynomial of $F(\xi)$ around the point $\xi=\xi_{0}$. In our case, we will use the command Series $[F[\xi],\{\xi$ $, 0, n\}]$ for $n \geq 3$, since we need at least four equations to determine the five unknowns $w, m, p$, and $\lambda$ in terms of the coefficients of Equation (33). Of course, the series method is applicable to solve the BBM when $n=1$. Our aim is to show two different methods. Let us proceed. We have that $F^{(2 n+1)}$ $\left(\xi_{0}\right)=0$ for all $n=0,1,2,3, \cdots$, and

$$
\begin{aligned}
F(\xi)= & F\left(\xi_{0}\right)+\frac{1}{2} F^{\prime \prime}\left(\xi_{0}\right)\left(\xi-\xi_{0}\right)^{2}+\frac{1}{24} F^{\prime \prime \prime \prime}\left(\xi_{0}\right)\left(\xi-\xi_{0}\right)^{4} \\
& +\frac{1}{720} F^{\prime \prime \prime \prime \prime \prime \prime}\left(\xi_{0}\right)\left(\xi-\xi_{0}\right)^{6}+\cdots,
\end{aligned}
$$

where

$$
\begin{aligned}
& F\left(\xi_{0}\right)=\frac{1}{12}\left(12 c_{1}+12_{2}+6 \lambda+\sigma\right)=0, \\
& \lambda=-\frac{1}{6}\left(\sigma+12 c_{1}+12 c_{2}\right), \\
& F^{\prime \prime}\left(\xi_{0}\right)=-\frac{(p-1) w\left(6 c_{1} p+3 p w \lambda \mu+6 p \lambda+2 p \sigma-3 w \lambda \mu\right)}{12 p^{2}}=0 \\
& \quad p=\frac{3 w \lambda \mu}{6 c_{1}+6 \lambda+3 w \lambda \mu+2 \sigma}, \\
& 012 p^{3} F^{\prime \prime \prime \prime}\left(\xi_{0}\right)=(p-1) w^{2} \\
& \quad \cdot\left(-18 c_{1} p+6 c_{1} p^{2}+24 c_{1} m p^{2}-27 p \lambda+15 p^{2} \lambda+24 m p^{2} \lambda\right. \\
& \quad+36 w \lambda \mu-48 p w \lambda \mu-48 m p w \lambda \mu+12 p^{2} w \lambda \mu \\
& \left.+48 m p^{2} w \lambda \mu-15 p \sigma+11 p^{2} \sigma+8 m p^{2} \sigma\right) \\
& w=-\frac{p\left(6 c_{1}(4 m p+p-3)+3(8 m p+5 p-9) \lambda+(8 m p+11 p-15) \sigma\right)}{12(p-1)(4 m p+p-3) \lambda \mu}
\end{aligned}
$$

$$
\begin{aligned}
0= & -12 p^{4} F^{\prime \prime \prime \prime \prime \prime \prime}\left(\xi_{0}\right)=(p-1) w^{3} \\
& \cdot\left[32 p^{2}\left(3 p \lambda-24 w \lambda \mu+24 p w \lambda \mu+p \sigma+3 p c_{1}\right) m^{2}\right. \\
& +4 p\left(-135 p \lambda+111 p^{2} \lambda+450 w \lambda \mu-708 p w \lambda \mu\right. \\
& \left.+258 p^{2} w \lambda \mu-75 p \sigma+67 p^{2} \sigma-90 p c_{1}+66 p^{2} c_{1}\right) m \\
& +270 p \lambda-270 p^{2} \lambda+51 p^{3} \lambda-675 w \lambda \mu+1125 p w \lambda \mu \\
& -498 p^{2} w \lambda \mu+48 p^{3} w \lambda \mu+225 p \sigma-300 p^{2} \sigma+92 p^{3} \sigma \\
& \left.+135 p c_{1},-90 p^{2} c_{1}+6 p^{3} c_{1}\right] .
\end{aligned}
$$

Solving this last equation for $m$ and taking into account (45), (47), and (49), we obtain after long algebraic calculations following solution:

$$
\begin{aligned}
& \lambda=\frac{1}{6}\left(-12 c_{1}-12 c_{2}-\sigma\right), \\
& w=\frac{2 \sqrt{6 \Delta}}{\mu\left(12 c_{1}+12 c_{2}+\sigma\right)}, \\
& p=-\frac{-6 c_{1}-24 c_{2}+\sqrt{6 \Delta}}{12 c_{2}+\sigma},
\end{aligned}
$$

$$
\begin{gathered}
m=\frac{216 c_{1} c_{2}+432 c_{2}^{2}+6 c_{1} \sigma-\sigma^{2}+\left(12 \sqrt{6} c_{2}-24 c_{2} \sigma+3 \sqrt{6} \sigma\right) \sqrt{\Delta}}{8\left(6 c_{1}+24 c_{2}-\sqrt{6 \Delta}\right)\left(6 c_{1}+12 c_{2}-\sigma\right)}, \\
\Delta=\left(c_{1}+4 c_{2}\right)\left(6 c_{1}+12 c_{2}-\sigma\right), \sigma=\sigma, \mu=\mu .
\end{gathered}
$$

Direct calculations show that function $u=u(\xi)$ defined by (39) with parameters given by (51)-(55) and (41) is a solution to Equation (41). Then, function $u=u\left(x-\lambda t-\xi_{0}\right)$ is a solution to the modified BBM equation

$$
u_{t}+\sigma u^{2} u_{x}-\mu u_{x x t}=0
$$

Observe that $\operatorname{cn}(x, 1)=\operatorname{sech}(x)$. It may be verified that the following function is a solution to Equation (56):

$$
u(x, t)=\alpha\left(1+\frac{\beta}{1+\gamma \cosh (k(x+\lambda t)+C)}\right),
$$

for the choice 


$$
\begin{aligned}
& c_{1}=-\frac{2 \alpha^{3} \gamma^{4} \sigma}{3\left(\gamma^{2}-2\right)^{3}}, \\
& c_{2}=\frac{\alpha^{4}(\gamma-2) \gamma^{6}(\gamma+2) \sigma}{12\left(\gamma^{2}-2\right)^{4}}, \\
& k=\frac{\sqrt{2} \sqrt{1-\gamma^{2}}}{\sqrt{\left(\gamma^{2}+2\right) \mu}} \\
& \lambda=-\frac{\alpha^{2} \gamma^{2}\left(\gamma^{2}+2\right) \sigma}{3\left(\gamma^{2}-2\right)^{2}}, \\
& \beta=-\frac{2(\gamma-1)(\gamma+1)}{\gamma^{2}-2} .
\end{aligned}
$$

We obtain soliton solutions for $\left(1-\gamma^{2}\right) \mu>0$ and trigonometric solutions for $\left(1-\gamma^{2}\right) \mu<0$.

2.3. Solution for a Generalized BBM Equation. Let us consider the following generalized BBM equation:

$$
u_{t}+a u_{x}+b u^{p} u_{x}-\delta u_{x x t}=0 .
$$

In order to obtain new solutions to Equation (59), we make the travelling wave transformation

$$
u(x, t)=v^{-2 / p}(\xi), \text { where } \xi=x-\lambda t+\xi_{0}
$$

Inserting (60) into (59) gives

$$
\begin{aligned}
& p v^{\prime}(\xi)(\xi)\left(p(a-\lambda) v(\xi)^{2}+b p-3 \delta \lambda(p+2) v(\xi) \mathrm{v}^{\prime \prime}(\xi)\right) \\
& \quad+\delta \lambda p^{2} v(\xi)^{2} v^{(3)}(\xi)+2 \delta \lambda(p+1)(p+2) \mathrm{v}^{\prime}(\xi)(\xi)^{3}=0
\end{aligned}
$$

Equation (61) is hard to integrate. We seek for an exact solution of the form

$$
v(\xi)=A+\frac{B}{1+C \wp\left(\xi ; g_{2}, g_{3}\right)} .
$$

Inserting ansatz (62) into (61) gives $R(\xi)=0$, where

$$
\begin{aligned}
R(\xi)= & c_{4} \wp\left(\xi ; g_{2}, g_{3}\right)^{4}+c_{3} \wp\left(\xi ; g_{2}, g_{3}\right)^{3}+c_{2} \wp\left(\xi ; g_{2}, g_{3}\right)^{2} \\
& +c_{1} \wp\left(\xi ; g_{2}, g_{3}\right)+c_{0},
\end{aligned}
$$

$$
\begin{aligned}
& c_{0}=2 a A^{2} p^{2}+4 a A B p^{2}+2 a B^{2} p^{2}-12 A^{2} C^{2} \delta g_{3} \lambda p^{2} \\
& +6 A^{2} C \delta g_{2} \lambda p^{2}-2 A^{2} \lambda p^{2}-12 A B C^{2} \delta g_{3} \lambda p^{2} \\
& +24 A B C^{2} \delta g_{3} \lambda p+9 A B C \delta g_{2} \lambda p^{2}-6 A B C \delta g_{2} \lambda p \\
& -4 A B \lambda p^{2}+2 b p^{2}-8 B^{2} C^{2} \delta g_{3} \lambda-4 B^{2} C^{2} \delta g_{3} \lambda p^{2} \\
& +12 B^{2} C^{2} \delta g_{3} \lambda p+3 B^{2} C \delta g_{2} \lambda p^{2}-6 B^{2} C \delta g_{2} \lambda p-2 B^{2} \lambda p^{2} \text {, } \\
& c_{1}=8 a A^{2} C p^{2}+12 a A B C p^{2}+4 a B^{2} C p^{2}-24 A^{2} C^{3} \delta g_{3} \lambda p^{2} \\
& +6 A^{2} C^{2} \delta g_{2} \lambda p^{2}-8 A^{2} C \lambda p^{2}+24 A^{2} \delta \lambda p^{2} \\
& -12 A B C^{3} \delta g_{3} \lambda p^{2}+24 A B C^{3} \delta g_{3} \lambda p+6 A B C^{2} \delta g_{2} \lambda p^{2} \\
& +12 A B C^{2} \delta g_{2} \lambda p-12 A B C \lambda p^{2}+48 A B \delta \lambda p^{2}+8 b C p^{2} \\
& -8 B^{2} C^{2} \delta g_{2} \lambda-B^{2} C^{2} \delta g_{2} \lambda p^{2}+6 B^{2} C^{2} \delta g_{2} \lambda p-4 B^{2} C \lambda p^{2} \\
& +24 B^{2} \delta \lambda p^{2} \\
& c_{2}=C p\left(12 a A^{2} C p+12 a A B C p+2 a B^{2} C p-12 A^{2} C^{3} \delta g_{3} \lambda p\right. \\
& -6 A^{2} C^{2} \delta g_{2} \lambda p-12 A^{2} C \lambda p+24 A^{2} \delta \lambda p+18 A B C^{2} \delta g_{2} \lambda \\
& -3 A B C^{2} \delta g_{2} \lambda p-12 A B C \lambda p+72 A B \delta \lambda+36 A B \delta \lambda p \\
& \left.+12 b C p-2 B^{2} C \lambda p+72 B^{2} \delta \lambda+12 B^{2} \delta \lambda p\right) \text {, } \\
& c_{3}=2 C^{2}\left(4 a A^{2} C p^{2}+2 a A B C p^{2}-3 A^{2} C^{2} \delta g_{2} \lambda p^{2}-4 A^{2} C \lambda p^{2}\right. \\
& -12 A^{2} \delta \lambda p^{2}-2 A B C \lambda p^{2}-12 A B \delta \lambda p^{2}+24 A B \delta \lambda p \\
& \left.+4 b C p^{2}+16 B^{2} \delta \lambda+2 B^{2} \delta \lambda p^{2}+12 B^{2} \delta \lambda p\right) \text {, } \\
& c_{4}=2 C^{3} p\left(a A^{2} C p-A^{2} C \lambda p-12 A^{2} \delta \lambda p-12 A B \delta \lambda-6 A B \delta \lambda p+b C p\right) .
\end{aligned}
$$

Equating to zero the coefficients of $\wp^{j}\left(\xi ; g_{2}, g_{3}\right)$ gives a nonlinear algebraic system. Solving it with the aid of Wolfram Mathematica, we obtain

$$
\begin{gathered}
B=-6 A, \\
C=-\frac{24 \delta\left(a A^{2} p^{2}+3 a A^{2} p+2 a A^{2}+2 b\right)}{b p^{2}}, \\
\lambda=\frac{a(p+2)(p+1) A^{2}+2 b}{A^{2}(p+1)(p+2)}, \\
g_{2}=\frac{b^{2} p^{4}}{48 \delta^{2}\left(a(p+2)(p+1) A^{2}+2 b\right)^{2}}, \\
g_{2}=-\frac{b^{3} p^{6}}{1728 \delta^{3}\left(a(p+2)(p+1) A^{2}+2 b\right)^{3}} .
\end{gathered}
$$

Other solutions may be obtained choosing $B$ from the condition

$$
\begin{aligned}
12 A^{2} p^{2}\left(30 a A^{2}+30 b+15 a A^{2} p+b p-b p^{2}\right) \\
-2 A p\left(28 a A^{2}+28 b-166 a A^{2} p-118 b p-88 a A^{2} p^{2}\right. \\
\left.-4 b p^{2}+a A^{2} p^{3}+4 b p^{3}\right) B-(p-1)(p+2) \\
\cdot\left(-24 a A^{2}-24 b-38 a A^{2} p-2 b p+a A^{2} p^{2}+b p^{2}\right) B^{2}=0 .
\end{aligned}
$$


Then, a solution to (61) is

$$
v(\xi)=A-\frac{6 A}{1-\left(24 \delta\left(a(p+2)(p+1) A^{2}+2 b\right) / b p^{2}\right) \wp\left(\xi ; g_{2}, g_{3}\right)},
$$

where $g_{2}, g_{3}$, and $\lambda$ are obtained from (65). The obtained solution is valid for

$$
A \delta b\left(a(p+2)(p+1) A^{2}+2 b\right) p(p+1)(p+2) \neq 0
$$

We have solved the generalized BBM equation for any $p$ such that $p(p+1)(p+2) \neq 0$. The exact traveling wave solution is given by

$$
u(x, t)=\left(A-\frac{6 A}{1-\left(24 \delta\left(a A^{2} p^{2}+3 a A^{2} p+2 a A^{2}+2 b\right) / b p^{2}\right) \wp\left(x-\left(a(p+2)(p+1) A^{2}+2 b / A^{2}(p+1)(p+2)\right) t+\xi_{0} ; g_{2}, g_{3}\right)}\right)^{-2 / p}
$$

The numbers $A$ and $\xi_{0}$ are arbitrary. Particular cases are

(1) $p=1$ :

$$
\left.u(x, t)=\left(A+\frac{6 A b}{48 \delta\left(3 a A^{2}+b\right) \wp\left(\left(b+3 A^{2} a / 3 A^{2}\right) t-x-\xi_{0} ; b^{2} / 192\left(3 a A^{2}+b\right)^{2} \delta^{2},-\left(b^{3} / 13824\left(3 a A^{2}+b\right)^{3} \delta^{3}\right)\right.}\right)^{-2}\right)^{-2}
$$

(2) $p=2$ :

$$
\left.u(x, t)=\left(A+\frac{6 A b}{12 \delta\left(6 a A^{2}+b\right) \wp\left(\left(b+6 A^{2} a / 6 A^{2}\right) t-x-\xi_{0} ; b^{2} / 12\left(6 a A^{2}+b\right)^{2} \delta^{2},-\left(b^{3} / 216\left(6 a A^{2}+b\right)^{3} \delta^{3}\right)\right.}\right)^{-b}\right)^{-1}
$$

(3) $p=1 / 2$ :

$$
\left.u(x, t)=\left(A+\frac{6 A b}{24 \delta\left(15 a A^{2}+8 b\right) \wp\left(\left(8 b+15 A^{2} a / 15 A^{2}\right) t-x-\xi_{0} ; b^{2} / 48\left(15 a A^{2}+8 b\right)^{2} \delta^{2},-\left(b^{3} / 1728\left(15 a A^{2}+8 b\right)^{3} \delta^{3}\right)\right.}\right)^{-b}\right)^{-4}
$$

2.4. Forbidden Values. Let us examine the cases when $p(p+$ 1) $(p+2)=0$.

Case $1(p=0)$. For this value, Equation (59) takes the form

$$
u_{t}+a u_{x}+b u_{x}-\delta u_{x x t}=0
$$

After doing a traveling wave transformation, we get the linear ode

$$
(a+b-\lambda) v^{\prime}(\xi)+\delta v^{\prime \prime \prime}(\xi)=0,
$$

whose general solution is given by

$$
v(\xi)=\frac{\sqrt{\delta \lambda}}{\sqrt{\lambda-a-b}}\left(c_{1} e^{(\sqrt{\lambda-a-b} / \sqrt{\delta \lambda}) \xi}-c_{2} e^{-(\sqrt{\lambda-a-b} / \sqrt{\delta \lambda}) \xi}\right)+c_{3} .
$$

Case $2((p+1)(p+2)=0)$. Making the traveling wave transformation $\xi=x-\lambda t+\xi_{0}$ and making use of the ansatz (62), we obtain that $B=0$, which is a trivial solution. So, in the cases $p=-1$ and $p=-2$, we cannot get nontrivial solutions to the generalized BBM equation. 


\section{Analysis and Discussion}

We obtained the traveling wave solutions to BBM equation $u_{t}+\sigma u u_{x}-\mu u_{x x t}=0$ and modified BBM equation $u_{t}+\sigma u^{2}$ $u_{x}-\mu u_{x x t}=0$. Other solutions were obtained in [7] using the extended Jacobi elliptic function expansion method. However, in our approach, we got nonzero integration constants. Most authors get zero integration constants to simplify matters, but this may cause loss of solutions. We also may consider the following combined BBM equation:

$$
u_{t}+\left(a u+\sigma u^{2}\right) u_{x}-\mu u_{x x t}=0
$$

Making the traveling wave transformation $u=v(\xi), \xi=$ $x-\lambda t+\xi_{0}$ gives

$$
-\lambda v^{\prime}(\xi)+\left(a v(\xi)+\sigma v^{2}(\xi)\right) v^{\prime}(\xi)+\delta \lambda v^{\prime \prime \prime}(\xi)=0
$$

We now integrate once with respect to $\xi$, and denoting the constant of integration by $c_{1}$, we obtain the ode

$$
v^{\prime \prime}(\xi)+\frac{c_{1}}{\delta \lambda}-\frac{1}{\delta} v(\xi)+\frac{a}{2 \delta \lambda} v(\xi)^{2}+\frac{\sigma}{3 \delta \lambda} v(\xi)^{3}=0
$$

This last equation is a Duffing-Helmholtz equation. The general form of the undamped and unforced DuffingHelmholtz equation reads

$$
v^{\prime \prime}(\xi)+n+p v(\xi)+q v^{2}(\xi)+r v^{3}(\xi)=0
$$

The general solution to Equation (79) may be expressed in the ansatz form

$$
v(\xi)=A+\frac{B}{1+C \wp\left(\xi+D ; g_{2}, g_{3}\right)} .
$$

Indeed, given the initial conditions

$$
\begin{gathered}
v(0)=v_{0}, \\
v^{\prime}(0)=\dot{v}_{0},
\end{gathered}
$$

the solution to the initial value problem (80)-(81) is given by (80), where

$$
\begin{aligned}
B= & -\frac{6\left(A^{3} r+A^{2} q+A p+n\right)}{3 A^{2} r+2 A q+p}, \\
C= & \frac{12}{3 A^{2} r+2 A q+p}, \\
g_{2}= & \frac{1}{12}\left(-3 A^{4} r^{2}-4 A^{3} q r-6 A^{2} p r-12 A n r-4 n q+p^{2}\right), \\
g_{3}= & \frac{1}{216}\left(9 A^{4} p r^{2}-3 A^{4} q^{2} r+12 A^{3} p q r-4 A^{3} q^{3}+18 A^{2} p^{2} r\right. \\
& \left.-6 A^{2} p q^{2}+36 A n p r-12 A n q^{2}+27 n^{2} r-6 n p q+p^{3}\right),
\end{aligned}
$$

$$
D= \pm \wp^{-1}\left(\frac{v_{0}-A-B}{C\left(A-v_{0}\right)} ; g_{2}, g_{3}\right)
$$

The number $A$ is a solution to the quartic

$3 r A^{4}+4 q A^{3}+6 p A^{2}+12 n A-\left(12 n v_{0}+6 p v_{0}^{2}+4 q v_{0}^{3}+3 r v_{0}^{4}+6 \dot{v}_{0}^{2}\right)=0$.

In soliton theory, we are interested in sech or tanh solutions to Equation (79). The soliton solutions may occur only when

$$
27 n r^{2}-9 p q r+2 q^{3}=0
$$

In this case, we will have the following soliton solution to Equation (79):

$$
v(\xi)=-\frac{q}{3 r}+\frac{3 A r+q}{3 r} \operatorname{sech}\left(\frac{3 A r+q}{3 \sqrt{2 r}} \xi\right), \text { for } 3 A r+q \neq 0
$$

Let us examine condition (85) for (78). We must have

$$
a^{3}+6 a \lambda \sigma+12 c_{1} \sigma^{2}=0
$$

Solving this equation for $c_{1}$, we will have the following soliton solution to equation (18):

$$
\begin{aligned}
u(x, t)= & -\frac{a}{2 \sigma}+\frac{a+2 A \sigma}{2 \sigma} \operatorname{sech}\left(\frac{a+2 A \sigma}{\sqrt{\delta\left(4 a A \sigma+4 A^{2} \sigma^{2}-5 a^{2}\right)}}\right. \\
& \left.\cdot\left(x+\frac{1}{24}\left(\frac{5 a^{2}}{\sigma}-4 a A-4 A^{2} \sigma\right) t+\xi_{0}\right)\right) .
\end{aligned}
$$

In (88), the constants $A$ and $\xi_{0}$ are arbitrary. In the case when $\sigma=0$, Equation (18) converts into usual BBM equation $u_{t}+a u_{x}-\mu u_{x x t}=0$.

In a more general fashion, we solved Equation (59). This equation was solved in $[2,5,8]$ using the substitution $u=v^{1 / p}$. In the present paper, we solved it by using the substitution $u=v^{-2 / p}$. Let us examine the odes obtained by means of these different substitutions. Using $u=v^{1 / p}$ gives

$$
\begin{aligned}
& n^{2} v(\xi)^{2}\left((a-\lambda) v^{\prime}(\xi)+\delta \lambda v^{(3)}(\xi)\right)+b n^{2} v(\xi)^{3} v^{\prime}(\xi) \\
& \quad+\delta \lambda(n-1)(2 n-1) v^{\prime}(\xi)^{3}-3 \delta \lambda(n-1) n v(\xi) v^{\prime}(\xi) v^{\prime \prime}(\xi)=0
\end{aligned}
$$

On the other hand, letting $u=v^{-2 / p}$ gives a different ode given by (61). So, our method gives other than that obtained in $[2,5,8]$ solutions.

The authors in [5] claim that there are no periodic solutions for the parameter values of different form $p=1, p=2$, 
or $p=1 / 2$. However, we obtained periodic solutions for $(p-1)(p-2)(p-1 / 2) \neq 0$.

On the other hand, we may solve the ode (89) by means of the ansatz (62). That was the approach the author employed in [8] by means of the substitution $u=v^{1 / p}$. Indeed, let us consider the equation [8]:

$$
u_{t}+u_{x}+a u^{p} u_{x}+u_{x x x}=0
$$

Let $u=v^{1 / p}\left(x-\lambda t+\xi_{0}\right)$. Then, the ode (90) converts into the ode

$$
\begin{aligned}
& -a p^{2} v(\xi)^{3} v^{\prime}(\xi)+p^{2} v(\xi)^{2}\left((\lambda-1) v^{\prime}(\xi)-v^{(3)}(\xi)\right) \\
& \quad+((3-2 p) p-1) v^{\prime}(\xi)^{3}+3(p-1) p v(\xi) v^{\prime}(\xi) v^{\prime \prime}(\xi)=0 .
\end{aligned}
$$

In order to solve equation (91), we will assume the ansatz form (62). Plugging this ansatz into (91), we get

$$
\begin{aligned}
d_{4} \wp\left(\xi ; g_{2}, g_{3}\right)^{4} & +d_{3} \wp\left(\xi ; g_{2}, g_{3}\right)^{3}+d_{2} \wp\left(\xi ; g_{2}, g_{3}\right)^{2} \\
& +d_{1} \wp\left(\xi ; g_{2}, g_{3}\right)+d_{0}=0,
\end{aligned}
$$

where

$$
\begin{aligned}
d_{4}= & 2 A C^{3} p\left(a A^{2} C p-A C \lambda p+A C p-12 A p-6 B p+6 B\right), \\
d_{3}= & 2 C^{2}\left(4 a A^{3} C p^{2}+3 a A^{2} B C p^{2}-3 A^{2} C^{2} g_{2} p^{2}-4 A^{2} C \lambda p^{2}\right. \\
& +4 A^{2} C p^{2}-12 A^{2} p^{2}-2 A B C \lambda p^{2}+2 A B C p^{2}-12 A B p^{2} \\
& \left.-12 A B p+2 B^{2} p^{2}-6 B^{2} p+4 B^{2}\right), \\
d_{2}= & C p\left(12 a A^{3} C p+18 a A^{2} B C p+6 a A B^{2} C p-12 A^{2} C^{3} g_{3} p\right. \\
& -6 A^{2} C^{2} g_{2} p-12 A^{2} C \lambda p+12 A^{2} C p+24 A^{2} p \\
& -3 A B C^{2} g_{2} p-9 A B C^{2} g_{2}-12 A B C \lambda p+12 A B C p \\
& \left.+36 A B p-36 A B-2 B^{2} C \lambda p+2 B^{2} C p+12 B^{2} p-36 B^{2}\right),
\end{aligned}
$$

$$
\begin{aligned}
d_{1}= & 8 a A^{3} C p^{2}+18 a A^{2} B C p^{2}+12 a A B^{2} C p^{2}+2 a B^{3} C p^{2} \\
& -24 A^{2} C^{3} g_{3} p^{2}+6 A^{2} C^{2} g_{2} p^{2}-8 A^{2} C \lambda p^{2}+8 A^{2} C p^{2} \\
& +24 A^{2} p^{2}-12 A B C^{3} g_{3} p^{2}-12 A B C^{3} g_{3} p+6 A B C^{2} g_{2} p^{2} \\
& -6 A B C^{2} g_{2} p-12 A B C \lambda p^{2}+12 A B C p^{2}+48 A B p^{2} \\
& -B^{2} C^{2} g_{2} p^{2}-3 B^{2} C^{2} g_{2} p-2 B^{2} C^{2} g_{2}-4 B^{2} C \lambda p^{2} \\
& +4 B^{2} C p^{2}+24 B^{2} p^{2},
\end{aligned}
$$

$$
\begin{aligned}
d_{0}= & 2 a A^{3} p^{2}+6 a A^{2} B p^{2}+6 a A B^{2} p^{2}+2 a B^{3} p^{2}-12 A^{2} C^{2} g_{3} p^{2} \\
& +6 A^{2} C g_{2} p^{2}-2 A^{2} \lambda p^{2}+2 A^{2} p^{2}-12 A B C^{2} g_{3} p^{2} \\
& -12 A B C^{2} g_{3} p+9 A B C g_{2} p^{2}+3 A B C g_{2} p-4 A B \lambda p^{2} \\
& +4 A B p^{2}-4 B^{2} C^{2} g_{3} p^{2}-6 B^{2} C^{2} g_{3} p-2 B^{2} C^{2} g_{3} \\
& +3 B^{2} C g_{2} p^{2}+3 B^{2} C g_{2} p-2 B^{2} \lambda p^{2}+2 B^{2} p^{2} .
\end{aligned}
$$

Solving the system $d_{0}=d_{1}=d_{2}=d_{3}=d_{4}=0$ gives the following solution:

$$
\begin{aligned}
B & =-\frac{3 A}{2}, \\
C & =\frac{3(p+1)(p+2)}{a A p^{2}}, \\
g_{2} & =\frac{a^{2} A^{2} p^{4}}{3(p+1)^{2}(p+2)^{2}}, \\
g_{3} & =-\frac{a^{3} A^{3} p^{6}}{27(p+1)^{3}(p+2)^{3}}, \\
\lambda & =\frac{2 a A+p^{2}+3 p+2}{(p+1)(p+2)} .
\end{aligned}
$$

Thus, a traveling wave solution to Equation (90) reads

$$
u(x, t)=\sqrt[p]{A-\frac{3 A / 2}{1+\left(3(p+1)(p+2) / a A p^{2}\right) \wp\left(x-\lambda t+\xi_{0} ; a^{2} A^{2} p^{4} / 3(p+1)^{2}(p+2)^{2},-\left(a^{3} A^{3} p^{6} / 27(p+1)^{3}(p+2)^{3}\right)\right)}} .
$$

In a similar way, we may consider the following more general equation than that considered in [2]:

$$
u_{t}+a u_{x}+b u^{p} u_{x}-\delta u_{x x t}+\gamma u_{x x t}=0 .
$$

In [9], the author solved the following generalization of the BBM equation:

$$
u_{t}+\alpha u_{x}+\left(\beta u^{n}+\gamma u^{2 n}\right) u_{x}-\delta u_{x x t}=0
$$

Let us consider the following more general variant of BBM equation:

$$
u_{t}+\alpha u_{x}+\left(\beta u^{n}+\gamma u^{2 n}\right) u_{x}-\delta u_{x x t}-\kappa u_{x x x}=0
$$

We again may use the ansatz (62) to solve this equation. Making the traveling wave transformation $u=v^{1 / p}(x-\lambda t+$ $\left.\xi_{0}\right)$ gives the ode 


$$
\begin{aligned}
& n^{2} v(\xi)^{2}\left(v^{\prime}(\xi)(\alpha-\lambda)+v^{\prime \prime \prime}(\xi)(\delta \lambda-\kappa)\right)+\beta n^{2} v^{\prime}(\xi) v(\xi)^{3} \\
& \quad+\gamma n^{2} v^{\prime}(\xi) v(\xi)^{4}+3(n-1) n v^{\prime}(\xi) v^{\prime \prime}(\xi) v(\xi)(\kappa-\delta \lambda) \\
& \quad+(1-n)(2 n-1) v^{\prime}(\xi)^{3}(\kappa-\delta \lambda)=0 .
\end{aligned}
$$

Plugging the ansatz (62) into the ode (99) and equating to zero the coefficients of $\wp^{j}$ will give a very large algebraic system. After some hard algebraic calculations, we get the following solution:

$$
\begin{gathered}
B=-\frac{6 A(A \gamma(n+2)+\beta+2 \beta n)}{5 A \gamma(n+2)+\beta(8 n+4)}, \\
C=\frac{12\left(A^{2} \gamma \delta(n+2)+2 A \beta \delta(2 n+1)+\alpha \delta\left(2 n^{3}+7 n^{2}+7 n+2\right)-\kappa\left(2 n^{3}+7 n^{2}+7 n+2\right)\right)}{A n^{2}(5 A \gamma(n+2)+\beta(8 n+4))}, \\
g_{2}=\frac{A^{2} n^{4}(A \gamma(n+2)+\beta(4 n+2))^{2}}{12\left(A^{2} \gamma \delta(n+2)+2 A \beta \delta(2 n+1)+\alpha \delta\left(2 n^{3}+7 n^{2}+7 n+2\right)-\kappa\left(2 n^{3}+7 n^{2}+7 n+2\right)\right)^{2}}, \\
\lambda=\frac{A^{3} n^{6}(A \gamma(n+2)+\beta(4 n+2))^{3}}{216\left(A^{2} \gamma \delta(n+2)+2 A \beta \delta(2 n+1)+\alpha \delta\left(2 n^{3}+7 n^{2}+7 n+2\right)-\kappa\left(2 n^{3}+7 n^{2}+7 n+2\right)\right)^{3}}, \\
\left(n(A \gamma(n+2)+\beta(4 n+2))+\alpha\left(2 n^{3}+7 n^{2}+7 n+2\right)\right.
\end{gathered}
$$

The forbidden values are $(n+1)(n+2)(2 n+1)=0$. The soliton solutions may be obtained under the condition

$$
(2 n-1)(n-2)=0 .
$$

For $n=2$, we have the following soliton solution to Equation (98):

$$
u(x, t)=\sqrt{-\frac{5 \beta}{8 \gamma} \pm \frac{5 \beta}{8 \gamma} \operatorname{sech}\left(2 \sqrt{5} \sqrt{\frac{\beta^{2}}{192 \alpha \gamma \delta-25 \beta^{2} \delta-192 \gamma \kappa}}\left(x-\frac{192 \alpha \gamma-25 \beta^{2}}{192 \gamma} t+\xi_{0}\right)\right)} .
$$

In the case when $n=1 / 2$, we have the following solution to Equation (98):

$$
\begin{aligned}
u(x, t)= & \left(-\frac{2 \beta}{5 \gamma} \pm \frac{2 \sqrt{2} \beta}{5 \gamma} \operatorname{sech}\left(\sqrt{\frac{2 \beta^{2}}{75 \alpha \gamma \delta-16 \beta^{2} \delta-75 \gamma \kappa}}\right.\right. \\
& \left.\left.\cdot\left(x-\frac{75 \alpha \gamma-16 \beta^{2}}{75 \gamma} t+\xi_{0}\right)\right)\right)^{2} .
\end{aligned}
$$

Finally, more solutions may be obtained by means of the transformation $u=v^{-2 / n}$. We will not derive them here.

\section{Conclusions}

We successfully obtained exact solutions to regularized long-wave and generalized BBM equation by using different approaches. We showed the way to derive all traveling wave solutions to all known until now variants of BBM. We compared the known previously published solutions with the solutions obtained in this work. Other methods to find exact solutions to nonlinear differential equations may be found in $[1-3,7,9-39]$.

\section{Data Availability}

Data statement is not applicable.

\section{Conflicts of Interest}

The authors declare that they have no conflicts of interest.

\section{Acknowledgments}

The authors aknowledge Dr. S.A. Tantawy at Al Baha University for additional comments.

\section{References}

[1] T. B. Benjamin, J. L. Bona, and J. J. Mahony, "Model equations for long waves in nonlinear dispersive systems," Philosophical Transactions of the Royal Society of London, Series A: Mathematical, Physical and Engineering Sciences, vol. 272, pp. 4778, 1972.

[2] P. G. Estevez, S. Kuru, J. Negro, and L. M. Nieto, “Travelling wave solutions of the generalized Benjamin-Bona-Mahony equation," Chaos, Solitons \& Fractals, vol. 40, no. 4, pp. 2031-2040, 2009.

[3] E. Yusufoglu and A. Bekir, "The tanh and the sine-cosine methods for exact solutions of the MBBM and the Vakhnenko equations," Chaos Solitons Fractals, vol. 38, no. 4, pp. 11261133, 2008.

[4] A. S. Alofi, "Extended Jacobi elliptic function expansion method for nonlinear Benjamin-Bona-Mahony equations," International Mathematical Forum, vol. 7, no. 53, pp. 2639$2649,2012$.

[5] J.-y. An and W.-g. Zhang, "Exact periodic solutions to generalized BBM equation and relevant conclusions," Acta Mathematicae Applicatae Sinica, English Series, vol. 22, no. 3, pp. 509516, 2006.

[6] D. Lu, A. R. Seadawy, and A. Ali, "Applications of exact traveling wave solutions of modified Liouville and the symmetric regularized long wave equations via two new techniques," Results in Physics, vol. 9, pp. 1403-1410, 2018.

[7] A. H. Salas, C. A. Gómez, and J. G. Escobar, "Exact solutions for the general fifth-order KdV equation by the extended tanh method," Journal of Mathematical Sciences: Advances and Applications, vol. 1, no. 2, pp. 305-310, 2008.

[8] J. Nickel, "Elliptic solutions to a generalized BBM equation," Physics Letters A, vol. 364, no. 3-4, pp. 221-226, 2007.

[9] D. B. Belobo and T. Das, "Solitary and Jacobi elliptic wave solutions of the generalized Benjamin-Bona- Mahony equation," Communications in Nonlinear Science and Numerical Simulation, vol. 48, pp. 270-277, 2017.

[10] A. Biswas, "1-soliton solution of Benjamin-Bona-Mahoney equation with dual-power law nonlinearity," Communications in Nonlinear Science and Numerical Simulation, vol. 15, no. 10, pp. 2744-2746, 2010. 
[11] B. A. Kupershmidt, "KdV6: an integrable system," Physics Letters $A$, vol. 372, no. 15, pp. 2634-2639, 2008.

[12] Y. Yao and Y. Zeng, "The bi-Hamiltonian structure and new solutions of KdV6 equation," Letters in Mathematical Physics, vol. 86, no. 2-3, pp. 193-208, 2008.

[13] V. K. Mel'nikov, "Integration of the Korteweg-de-Vries equation with a source," Inverse Problems, vol. 6, pp. 233-246, 1990.

[14] E. Fan and Y. C. Hona, "Generalized tanh method extended to special types of nonlinear equations," Zeitschrift für Naturforschung A, vol. 57, no. 8, pp. 692-700, 2002.

[15] A. M. Wazwaz, "The extended tanh method for new solitons solutions for many forms of the fifth-order KdV equations," Applied Mathematics and Computation, vol. 184, no. 2, pp. 1002-1014, 2007.

[16] S. Zhang, "Exp-function method exactly solving a KdV equation with forcing term," Applied Mathematics and Computation, vol. 197, no. 1, pp. 128-134, 2008.

[17] J. H. He and L. N. Zhang, "Generalized solitary solution and compacton-like solution of the Jaulent- Miodek equations using the Exp-function method," Physics Letters A, vol. 372, no. 7, pp. 1044-1047, 2008.

[18] J. H. He, “An elementary introduction TO recently developed asymptotic methods and nanomechanics in textile engineering," International Journal of Modern Physics B, vol. 22, no. 21, pp. 3487-3578, 2008.

[19] R. Conte and M. Musette, "Link between solitary waves and projective Riccati equations," Journal of Physics A: Mathematical and General, vol. 25, no. 21, pp. 5609-5623, 1992.

[20] Z. Yan, "The Riccati equation with variable coefficients expansion algorithm to find more exact solutions of nonlinear differential equations," Computer Physics Communications, vol. 152, no. 1, pp. 1-8, 2003.

[21] E. Yomba, "The general projective Riccati equations method and exact solutions for class of nonlinear partial differential equations," Chinese Journal of Physics, vol. 436, pp. 991$1003,2005$.

[22] Y. Shang, Y. Huang, and W. Yuan, "New exact traveling wave solutions for the Klein-Gordon-Zakharov equations," Computers \& Mathematics with Applications, vol. 56, no. 5, pp. 1441-1450, 2008.

[23] M. A. Noor and S. T. Mohyud-Din, "Variational iteration method for solving higher-order nonlinear boundary value problems using Hes polynimials," International Journal of Nonlinear Sciences and Numerical Simulation, vol. 9, no. 2, pp. 141-157, 2008.

[24] J. H. He and H. Wu, "Construction of solitary solution and compacton-like solution by variational iteration method," Chaos Solitons \& Fractals, vol. 29, no. 1, pp. 108-113, 2006.

[25] S. T. Mohyud-Din, M. A. Noor, and K. I. Noor, "Travelling wave solutions of seventh-order generalized $\mathrm{KdV}$ equations using Hes polynomials," Communications in Nonlinear Science and Numerical Simulation, vol. 10, no. 2, pp. 223-229, 2009.

[26] S. T. Mohyud-Din and M. A. Noor, "Homotopy perturbation method for solving partial differential equations," Zeitschrift für Naturforschung A, vol. 64, no. 3-4, pp. 157-170, 2009.

[27] H. Mirgolbabai, D. D. Ganji, and H. Taherian, "Soliton solution of the Kadomtse-Petviashvili equation by homotopy perturbation method," World Journal of Modeling and Simulation, vol. 51, pp. 38-44, 2009.
[28] H. Mirgolbabai and D. D. Ganji, "Application of homotopy perturbation method to the combined KdV-mKdV equation," Journal of Applied Sciences, vol. 919, pp. 3587-3592, 2009.

[29] H. Mirgolbabai, A. Barari, and G. Domiri, "Analytical solution of forced-convective boundary-layer flow over a flat plate," Archives of Civil and Mechanical Engineering, vol. 10, no. 2, pp. 41-51, 2010.

[30] S. T. Mohyud-Din, M. A. Noor, and K. I. Noor, "Some Relatively New Techniques for Nonlinear Problems," Mathematical Problems in Engineering, vol. 2009, Article ID 234849, 25 pages, 2009.

[31] S. T. Mohyud-Din, M. A. Noor, and K. I. Noor, "Traveling wave solutions of seventh-order generalized $\mathrm{KdV}$ equations by variational iteration method using Adomian's polynomials," International Journal of Modern Physics B, vol. 23, no. 15, pp. 3265-3277, 2009.

[32] J. H. He, "Some asymptotic methods for strongly nonlinear equations," International journal of Modern physics B, vol. 20, no. 10, pp. 1141-1199, 2006.

[33] A. M. Wazwaz, "The integrable KdV6 equations: Multiple soliton solutions and multiple singular soliton solutions," Applied Mathematics and Computation, vol. 204, no. 2, pp. 963-972, 2008.

[34] Y. Zhang, X. Cai, and H. Xu, "A note on "The integrable KdV6 equation: multiple soliton solutions and multiple singular soliton solutions"," Mathematics of Computation, vol. 214, no. 1, pp. 1-3, 2009.

[35] A. H. Salas, "Symbolic computation of solutions for a forced burgers equation," Applied Mathematics and Computation, vol. 216, no. 1, pp. 18-26, 2010.

[36] A. H. Salas S and C. A. Gómez S, "Exact solutions for a thirdorder $\mathrm{KdV}$ equation with variable coefficients and forcing term," Mathematical Problems in Engineering, vol. 2009, Article ID 737928, 13 pages, 2009.

[37] A. H. Salas, "Some solutions for a type of generalized SawadaKotera equation," Applied Mathematics and Computation, vol. 196, no. 2, pp. 812-817, 2008.

[38] A. H. Salas, "Exact solutions for the general fifth KdV equation by the exp function method," Applied Mathematics and Computation, vol. 205, no. 1, pp. 291-297, 2008.

[39] A. H. Salas and C. A. Gómez S, “Application of the Cole-Hopf Transformation for Finding Exact Solutions to Several Forms of the Seventh-Order KdV Equation," Mathematical Problems in Engineering, vol. 2010, Article ID 194329, 14 pages, 2010. 